\title{
Phonons near Peierls Structural Transition in Quasi-One-Dimensional Organic Crystals of TTF-TCNQ
}

\author{
Silvia Andronic, Anatolie Casian \\ Department of Computers, Informatics and Microelectronics, Technical University of Moldova, \\ Chisinau, Moldova \\ Email: acasian@mail.utm.md
}

Received 16 March 2016; accepted 19 April 2016; published 22 April 2016

Copyright (C) 2016 by authors and Scientific Research Publishing Inc. This work is licensed under the Creative Commons Attribution International License (CC BY). http://creativecommons.org/licenses/by/4.0/

(c) (i) Open Access

\begin{abstract}
The Peierls structural transition in quasi-one-dimensional organic crystals of TTF-TCNQ is investigated in the frame of a more complete physical model. The two most important electron-phonon interaction mechanisms are taken into account simultaneously. One is similar of that of deformation potential and the other is of polaron type. For simplicity, the $2 \mathrm{D}$ crystal model is considered. The renormalized phonon spectrum and the phonon polarization operator are calculated in the random phase approximation for different temperatures. The effects of interchain interaction on renormalized acoustic phonons and on the Peierls critical temperature are analyzed.
\end{abstract}

\section{Keywords}

Quasi-One-Dimensional Organic Crystals, TTF-TCNQ, Peierls Transition, Interchain Interaction, Renormalized Phonons

\section{Introduction}

Quasi-one-dimensional (Q1D) organic materials have received much interest during the last years due to more diverse and often unusual properties manifested by them [1]-[3]. Besides, their properties can be easily manipulated and controlled by molecular chemistry methods. It has been also mentioned that the highly conducting Q1D organic crystals may have very prospective thermoelectric applications [4] [5]. The charge transfer compound TTF-TCNQ (tetrathiafulvalene-tetracyanoquinodimethane) is the most investigated Q1D organic crystal with the high electrical conductivity [6]. Its structure consists of segregated chains or stacks of TTF and TCNQ molecules. The metallic properties are due to partial transfer of electrons from TTF to TCNQ molecules. 
With the decrease of temperature, two processes undergo. From one side, the lattice distortion is favorable because it diminishes the electronic energy of the crystal, lowering the Fermi energy. From other side, the lattice distortion increases the elastic lattice energy. At some temperature, when the first process prevails over the second one, a Peierls structural transition undergoes.

It is established that in TTF-TCNQ at $54 \mathrm{~K}$ the Peierls transition takes place into interacting TCNQ stacks with the opening of a band gap in the electronic spectrum above the Fermi energy and a strong reducing of electrical conductivity. Although the development of the pre-transitional 1D structural fluctuations is observed on a large temperature range below $150 \mathrm{~K}$ [7] [8], these fluctuations lead to modification of the phonon spectrum with the lowering temperature [9] [10]. If at some temperature, the renormalized phonon frequency becomes equal to zero, a periodic lattice distortion with the respective wave vector occurs and the Peierls structural transition is observed.

In the last time, the physical model of Q1D organic crystals was completed by an additional electron-phonon interaction mechanism. It takes into account the fluctuations due to acoustic longitudinal phonons of the polarization energy of molecules surrounding the conduction electron [11]-[13]. The molecules of TCNQ are relatively big and their polarizability is large too.

In [14], the Peierls structural transition in Q1D crystals of TTF-TCNQ type was investigated in this more complete physical model of the crystal, but in strictly $1 \mathrm{D}$ approximation. It was considered the case when the conduction band is half filled and Fermi dimensionless quasi momentum, $k_{\mathrm{F}}=\pi / 2$. The Peierls critical temperature was determined.

In [15], the Peierls transition has been studied also in the 1D physical model. But now the renormalized acoustic phonon frequencies $\Omega(q)$ as functions of wave number $q$ were calculated: 1 ) when the conduction band is half filled and the dimensionless Fermi momentum $k_{\mathrm{F}}=\pi / 2$ and 2) when the concentration of conduction electrons is reduced and the band is filled up to a quarter of the Brillouin zone, $k_{\mathrm{F}}=\pi / 4$. It was shown that a more detailed calculation considerably modifies the dependences $\Omega(q)$. In both cases, the critical temperature of transition was determined.

The 2D physical model for the real crystals of TTF-TCNQ was investigated in [16]. The polarization operator as a function of temperature was calculated numerically for different values of the parameter $d$, which describes the ratio of the transfer energy in the direction transversal to conductive chains to the transfer energy along the chains. The Peierls critical temperature was determined in two cases: 1 ) when the dimensionless Fermi momentum is $k_{\mathrm{F}}=0.59 \pi / 2$, and 2) when the carrier' concentration is slightly modified and the Fermi momentum $k_{\mathrm{F}}=$ $0.59 \pi / 2 \pm \delta$, where $\delta$ represents the variation of the Fermi momentum. In this paper, the numerical modellings were performed for the values of the sound velocity at low temperatures taken from [17]: $v_{s 1}=3.4 \times 10^{5} \mathrm{~cm} / \mathrm{s}$ along chains and $v_{\mathrm{s} 2}=5.25 \times 10^{5} \mathrm{~cm} / \mathrm{s}$ in transversal direction. However, more exact calculations of the Peierls critical temperature in TCNQ chains have shown that in order to achieve the experimental result, it is necessary to choose $v_{s 2}=0.5 v_{s 1}$. The last value has been used later on.

The aim of this paper is to present a detailed modeling of the Peierls transition in TTF-TCNQ crystals in the frame of above mentioned more complete physical model. The effects of interchain interaction on the dispersion of renormalized phonons and on Peierls critical temperature are analyzed. For the simplicity, we consider the 2D approximation.

\section{The Physical Model in 2D Approximation}

The structure of TTF-TCNQ crystal is described in many papers (see [2] and references therein). This compound forms Q1D organic crystals that are composed of TCNQ and TTF linear segregated chains. The TTF molecules are donors and TCNQ molecules are strong acceptors. Because the width of band energy in the TCNQ chains is much larger than that of TTF chains, we have neglected the latter in the first approximation. Thus, the crystal is composed only of TCNQ chains with sufficiently large conduction band that permit to neglect in the first approximation the Coulomb interaction. As a result, our treatment is valid only for temperature $T \geq 54 \mathrm{~K}$. For lower temperature it is necessary to take into account the TTF chains and the Coulomb interaction. The particularity of this structure is that TCNQ chains form parallel planar stacks with lattice constants $a=12.30 \AA$ between the adjacent chains and $b=3.82 \AA$ between the neighbor TCNQ molecules along the chain. The two-dimensional physical model described in [13] is applied considering the small interchain interaction in the plane of stacks.

The Hamiltonian of the 2D crystal in the tight binding and nearest neighbor approximations has the form: 


$$
H=\sum_{\boldsymbol{k}} \varepsilon(\boldsymbol{k}) a_{\boldsymbol{k}}^{+} a_{\boldsymbol{k}}+\sum_{q} \hbar \omega_{q} b_{q}^{+} b_{q}+\sum_{\boldsymbol{k}, \boldsymbol{q}} A(\boldsymbol{k}, \boldsymbol{q}) a_{\boldsymbol{k}}^{+} a_{\boldsymbol{k}-\boldsymbol{q}}\left(b_{q}+b_{-q}^{+}\right) .
$$

In Equation (1) the first term represents the energy operator of free electrons in the periodic field of the lattice, $a_{\boldsymbol{k}}^{+}\left(a_{\boldsymbol{k}}\right)$ are the creation and annihilation operators of such electron, where $\boldsymbol{k}$ is two-dimensional wave vector with projections $\left(k_{x}, k_{y}\right)$. The carrier energy $\varepsilon(\boldsymbol{k})$ is measured from the bottom of conduction band.

$$
\varepsilon(\boldsymbol{k})=2 w_{1}\left(1-\cos k_{x} b\right)-2 w_{2}\left(1-\cos k_{y} a\right)
$$

where $w_{1}$ and $w_{2}$ are the transfer energies of a carrier from one molecule to another along the chain ( $x$ direction) and in perpendicular direction ( $y$ direction). In Equation (1) $b_{q}^{+}\left(b_{q}\right)$ are creation and annihilation operators of an acoustic phonon with two-dimensional wave vector $\boldsymbol{q}$ and frequency $\omega_{q}$. The second term in the Equation (1) is the energy of longitudinal acoustic phonons

$$
\omega_{q}^{2}=\omega_{1}^{2} \sin ^{2}\left(q_{x} b / 2\right)+\omega_{2}^{2} \sin ^{2}\left(q_{y} a / 2\right)
$$

where $\omega_{1}$ and $\omega_{2}$ are limit frequencies for oscillations in $x$ and $y$ directions. The third term in Equation (1) represents the electron-phonon interactions. Two interaction mechanisms are considered. The first interaction is determined by the fluctuations of transfer energies $w_{1}$ and $w_{2}$, due to the intermolecular vibrations (acoustic phonons). This interaction is similar to that of deformation potential, and the coupling constants are proportional to the derivatives $w_{1}^{\prime}$ and $w_{2}^{\prime}$ of $w_{1}$ and $w_{2}$ with respect to the intermolecular distances, $w_{1}^{\prime}>0, w_{2}^{\prime}>0$. The second interaction is of polaron type. This interaction is conditioned by the fluctuations of the polarization energy of the molecules surrounding the conduction electron. The coupling constant of this interaction is proportional to the average polarizability of the molecule $\alpha_{0}$. This interaction is important for crystals composed of large molecules such as TCNQ, so as $\alpha_{0}$ is roughly proportional to the volume of molecule.

The square module of matrix element $A(\boldsymbol{k}, \boldsymbol{q})$ from Equation (1) has the following form:

$$
\begin{aligned}
|A(\boldsymbol{k}, \boldsymbol{q})|^{2}= & 2 \hbar w_{1}^{\prime 2}\left(N M \omega_{\boldsymbol{q}}\right)\left\{\left[\sin \left(k_{x} b\right)-\sin \left(k_{x}-q_{x}, b\right)+\gamma_{1} \sin \left(q_{x} b\right)\right]^{2}\right. \\
& \left.+d^{2}\left[\sin \left(k_{y} a\right)-\sin \left(k_{y}-q_{y}, a\right)+\gamma_{2} \sin \left(q_{y} a\right)\right]^{2}\right\}
\end{aligned}
$$

where $N$ is the number of molecules in the basic region of the crystal, $M$ is the mass of the molecule; $d=w_{2} / w_{1}=w_{2}^{\prime} / w_{1}^{\prime}$, parameters $\gamma_{1}$ and $\gamma_{2}$ have the sense of the amplitudes ratio of second electron-phonon interaction to the first one along chains and in transversal direction

$$
\gamma_{1}=2 e^{2} \alpha_{0} / b^{5} w_{1}^{\prime} ; \gamma_{2}=2 e^{2} \alpha_{0} / a^{5} w_{2}^{\prime} \text {. }
$$

From exact series of perturbation theory for the phonon Green function we sum up the diagrams containing $0,1,2, \cdots, \infty$ closed loops of two electron Green functions that make the most important contribution. This is the random phase approximation. We obtain for the Fourier component of the phonon Green function $D(\boldsymbol{q}, \Omega)$ :

$$
D(\boldsymbol{q}, \Omega)=D_{0}(\boldsymbol{q}, \Omega)-D_{0}(\boldsymbol{q}, \Omega) \Pi(\boldsymbol{q}, \Omega) D(\boldsymbol{q}, \Omega)
$$

where

$$
D_{0}(\boldsymbol{q}, \Omega)=\frac{1}{2}\left(\frac{1}{\Omega-\omega_{q}+i \delta}-\frac{1}{\Omega+\omega_{q}-i \delta}\right)
$$

is the free phonon Green function, $\delta \rightarrow 0+$ and $\Omega(\boldsymbol{q})$ is the renormalized phonon frequency. $\Pi(\boldsymbol{q}, \Omega)$ is the phonon polarization operator, $\boldsymbol{q}$ is the wave vector of longitudinal acoustic phonons. The real part of the dimensionless polarization operator $\operatorname{Re} \bar{\Pi}(\boldsymbol{q}, \Omega)$ is presented in the form:

$$
\operatorname{Re} \bar{\Pi}(\boldsymbol{q}, \Omega)=-\frac{\bar{N}}{\pi^{2} \hbar \omega_{\boldsymbol{q}}} \int_{-\pi}^{\pi} \mathrm{d} k_{x} \int_{-\pi}^{\pi} \mathrm{d} k_{y}|A(\boldsymbol{k}, \boldsymbol{q})|^{2} \frac{n_{\boldsymbol{k}}-n_{\boldsymbol{k}+\boldsymbol{q}}}{\varepsilon(\boldsymbol{k})-\varepsilon(\boldsymbol{k}+\boldsymbol{q})+\hbar \Omega} .
$$


Here, $\bar{N}$ is the number of elementary cells in the basic region of the crystal, the number of molecules is $N=r \bar{N}$, where $r=2$ is the number of molecules in the elementary cell, $\hbar$ is the Planck constant. The temperature enters in our expressions only through the Fermi distribution functions $n_{\boldsymbol{k}}$ and $n_{\boldsymbol{k}+\boldsymbol{q}} . \Omega(\boldsymbol{q})$ is determined by the pole of function $D(\boldsymbol{q}, \Omega)$ and is obtained from the transcendent dispersion equation

$$
\Omega(\boldsymbol{q})=\omega_{\boldsymbol{q}}[1-\bar{\Pi}(\boldsymbol{q}, \Omega)]^{1 / 2} .
$$

This equation can be solved only numerically.

\section{Results and Discussions}

The calculations were performed for the following parameters: $w_{1}=0.125 \mathrm{eV}, w_{1}^{\prime}=0.2 \mathrm{eV} \cdot \AA^{-1}, a=12.30 \AA, b$ $=3.82 \AA, k_{\mathrm{F}}=0.59 \pi / 2[1]$ [2], $v_{s 1}=3.4 \times 10^{5} \mathrm{~cm} / \mathrm{s}, v_{\mathrm{s} 2}=0.5 v_{s 1} \mathrm{~cm} / \mathrm{s}, d=0.015, M=3.7 \times 10^{5} \mathrm{~m}_{e}\left(m_{e}\right.$ is the mass of the free electron), $\gamma_{1}=1.37, r=2$. The parameter $\gamma_{2}$ is determined from the relation: $\gamma_{2}=\gamma_{1} 2^{5} b^{5} /\left(a^{5} d\right)$.

Figure 1 and Figure 2 show the dependences of renormalized phonon frequencies $\Omega\left(q_{x}\right)$ as functions of $q_{x}$ for different temperatures and different values of $q_{y}$. The same dependences for initial phonon frequency $\omega\left(q_{x}\right)$ are presented too. It is seen that the values of $\Omega\left(q_{x}\right)$ are diminished in comparison with those of frequency $\omega\left(q_{x}\right)$ in the absence of electron-phonon interaction. This means that the electron-phonon interaction diminishes the values of lattice elastic constants. In addition, it is observed that with a decrease of temperature $T$ the curves change their form, and in dependencies $\Omega\left(q_{x}\right)$ a minimum appears. This minimum becomes more pronounced at lower temperatures. After all, at certain temperature $\Omega\left(q_{x}\right)$ attains zero for $q_{x}=0.58 \pi$. At this temperature, the structural Peierls transition takes place. The deviation of $\Omega\left(q_{x}\right)=0$ from $q_{x}=2 k_{\mathrm{F}}$ is caused by $T \neq 0$ and $k_{\mathrm{F}} \neq \pi / 2$.

Figure 1(a) shows the case, when $q_{y}=0$. At $T=59.7 \mathrm{~K}$ the transition occurs in TCNQ chains alone. The crystal lattice along TCNQ chains changes from the initial state with the lattice constant $b$ to a new crystalline state with constant $4 b$, that is four times larger. At this temperature, the electrical conductivity is strongly diminished, so as a gap in the carrier spectrum is fully opened just above the Fermi energy. In addition, it is seen that the slope of $\Omega\left(q_{x}\right)$ at small $q_{x}$ is diminished in comparison with that of $\omega\left(q_{x}\right)$. This means that the electron-phonon interaction has reduced also the sound velocity in a large temperature interval.

When the interaction between TCNQ chains is taken into account $\left(q_{y} \neq 0\right)$, the Peierls critical temperature is diminished. Figure 1(b) and Figure 2 correspond to 2D physical model, $q_{y} \neq 0$. Figure 1 (b) shows $\Omega\left(q_{x}\right)$ for $q_{y}$ $=\pi / 4$ and different temperatures. One can observe that $\Omega\left(q_{x}\right)$ attains zero at $T \sim 59 \mathrm{~K}$, i.e. the transition takes place at this $T$.

When $q_{y}=2 k_{\mathrm{F}}$ (Figure 2(a)), the Peierls critical temperature some more decreases and has a value of $T \sim 56$ K. Figure 2(b) shows the dependences of $\Omega\left(q_{x}\right)$ on $q_{x}$ for $q_{y}=\pi$ and different temperatures. It is observed that the transition temperature decreases still more and equals $T \sim 54 \mathrm{~K}$. Note that this value agrees with the experimental data.

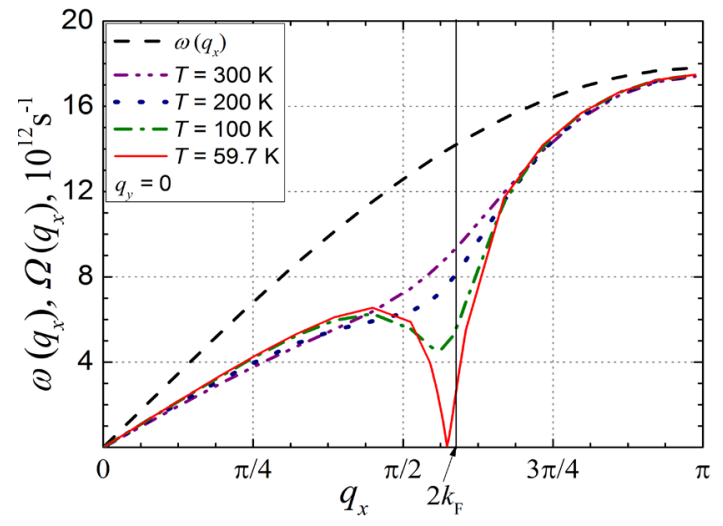

(a)

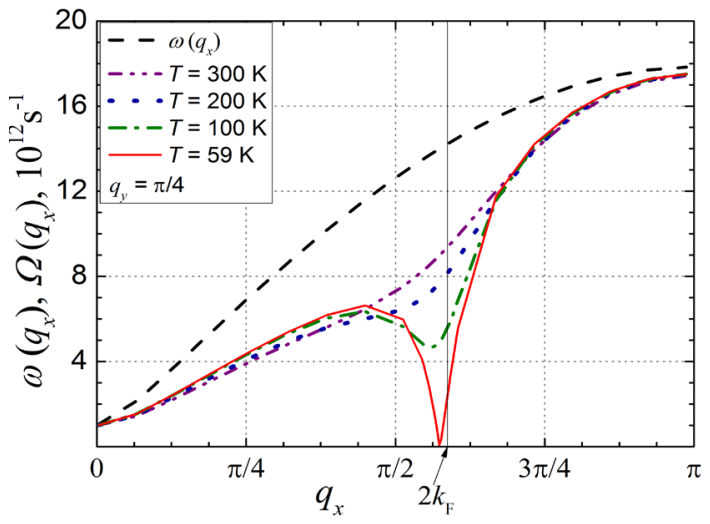

(b)

Figure 1. Renormalized phonon spectrum $\Omega\left(q_{x}\right)$ for $\gamma_{1}=1.37$ and different temperatures. The dashed line is for the spectrum of free phonons. (a) $q_{y}=0$; (b) $q_{y}=\pi / 4$. 
Figure 3 shows clearly that with the increase of parameter $q_{y}$, the Peierls critical temperature decrease. So, for $q_{y}=0, T \sim 59.7 \mathrm{~K}$; for $q_{y}=\pi / 4, T \sim 59 \mathrm{~K}$; for $q_{y}=2 k_{\mathrm{F}}, T \sim 56 \mathrm{~K}$ and for $q_{y}=\pi, T \sim 54 \mathrm{~K}$. This means that Peierls transition begins at $T \sim 59 \div 60 \mathrm{~K}$ in TCNQ chains alone, but when the interaction between TCNQ chains is taken into account in 2D approximation, Peierls transition occurs completely at $T \sim 54 \mathrm{~K}$. It is expected that 3D approximation will not introduce important modifications.

Figure 4 shows the dependencies of the real part of dimensionless polarization operator $\operatorname{Re} \bar{\Pi}\left(q_{x}, \Omega\right)$ as function of $q_{x}$ for different values of $q_{y}$ and different temperatures at $\Omega=0$. From the both graphs it is observed a very sharp peak near the value of unity.

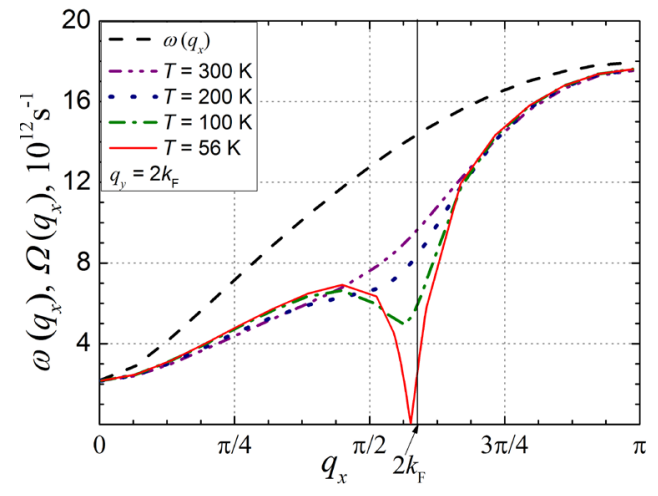

(a)

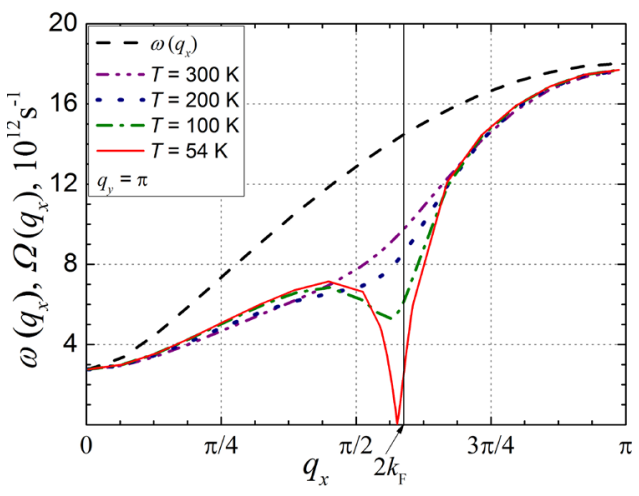

(b)

Figure 2. Renormalized phonon spectrum $\Omega\left(q_{x}\right)$ for $\gamma_{1}=1.37$ and different temperatures. The dashed line is for the spectrum of free phonons. (a) $q_{y}=2 k_{\mathrm{F}}$; (b) $q_{y}=\pi$.

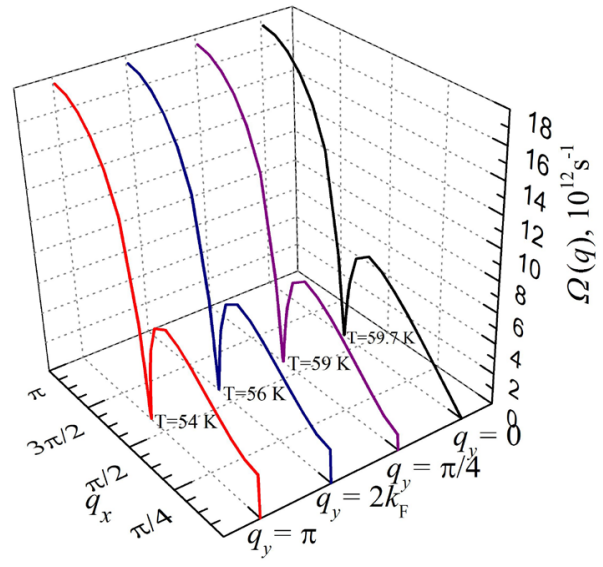

Figure 3. Renormalized phonon spectrum $\Omega\left(q_{x}\right)$ for $\gamma_{1}=1.37$, different values of $q_{y}$ and different temperatures.

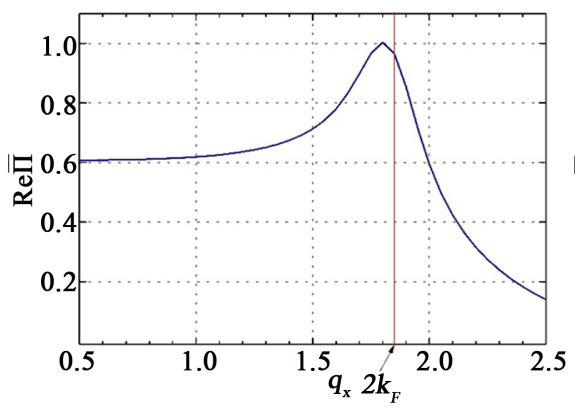

(a)

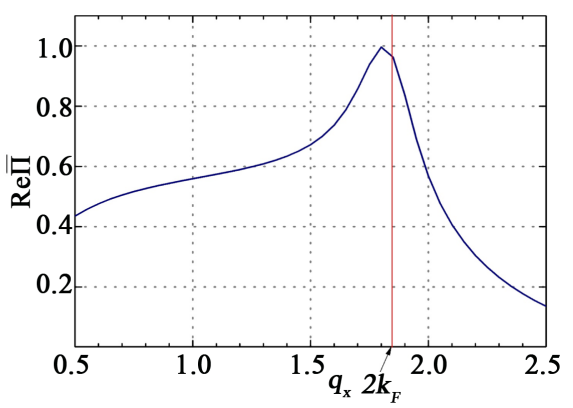

(b)

Figure 4. Polarization operator as function of $q_{x}$ for: (a) $q_{y}=0$ and $T=59.7 \mathrm{~K}$; (b) $q_{y}=\pi$ and $T=54 \mathrm{~K}$. 
Note that in above calculations we have used the value of $v_{s 1}=3.4 \times 10^{5} \mathrm{~cm} / \mathrm{s}$ at low temperatures from [17], but for $v_{s 2}$ we have taken $0.5 v_{s 1}$ instead of $v_{s 2}=5.25 \times 10^{5} \mathrm{~cm} / \mathrm{s}$ measured in [17]. If the last value of $v_{s 2}$ is applied, it results that a complete Peierls transition should undergo at $T=15 \mathrm{~K}$. Such big deviation of critical temperature from the observed one at $T=54 \mathrm{~K}$ suggests, that the sound velocity in the transversal direction cannot be larger than in longitudinal to chains direction and the most probably $v_{s 2} \sim 0.5 v_{s 1}$, as demonstrate our calculations of transition temperature. In addition, due to the quasi-one-dimensionality of the crystal, the chemical bonds in transversal direction must be weaken. This fact also suggests that $v_{s 2}$ should be less than $v_{s 1}$.

\section{Conclusion}

The behavior of phonons near Peierls transition has been studied in quasi-one-dimensional organic crystals of TTF-TCNQ in 2D approximation. A more complete crystal model is applied which takes into account the two most important electron-phonon interactions. One of them is of the deformation potential type. The coupling constants are proportional to the derivatives of the transfer energies $w_{1}$ and $w_{2}$ with respect to the intermolecular distances. The other interaction is similar to that of a polaron with the coupling constant proportional to the average polarizability of the molecule $\alpha_{0}$. The ratios of amplitudes of second electron-phonon interaction to the first one along chains and in transversal direction are noted by $\gamma_{1}$ and $\gamma_{2}$, respectively. Analytical expression for the polarization operator was obtained in random phase approximation. The numerical calculations for renormalized phonon spectrum, $\Omega\left(q_{x}\right)$, for different temperatures are presented: 1$)$ when $q_{y}=0$ and the interaction between TCNQ chains is neglected and 2) when $q_{y} \neq 0$ and interactions between the adjacent chains are considered. It has been established that Peierls transition begins at $T \sim 59.7 \mathrm{~K}$ in TCNQ chains alone and reduces considerably the electrical conductivity. Due to interchain interaction, the transition is finished at $T \sim 54 \mathrm{~K}$. It is demonstrated that the electron-phonon interaction diminishes $\Omega\left(q_{x}\right)$ with respect to initial frequency $\omega\left(q_{x}\right)$ and reduces the sound velocity in a large temperature interval.

\section{References}

[1] Jerome, D. (2004) Organic Conductors: From Charge Density Wave TTF-TCNQ to Superconducting (TMTSF) 2 PF6. Chemical Reviews, 104, 5565-5592. http://dx.doi.org/10.1021/cr030652g

[2] Jerome, D. (2012) Organic Superconductors: When Correlations and Magnetism Walk. Journal of Superconductivity and Novel Magnetism, 25, 633.

[3] Pouget, J.P. (2012) Bond and Charge Ordering in Low-Dimensional Organic Conductors. Physica B, 407, $1762-1770$. http://dx.doi.org/10.1016/j.physb.2012.01.025

[4] Casian, A. (2006) Thermoelectric Properties of Electrically Conducting Organic Materials. In: Rowe, D.M., Ed., Thermoelectric Handbook, Macro to Nano, Chap. 36, CRC Press, Boca Raton, FL, 36-1-36-8.

[5] Casian, A.I., Pflaum, J. and Sanduleac, I.I. (2015) Prospects of Low Dimensional Organic Materials for Thermoelectric Applications. Journal of Thermoelectricity, 1, 16.

[6] Chernenkaya, A., et al. (2015) Nature of the Empty States and Signature of the Charge Density Wave Instability and Upper Peierls Transition of TTF-TCNQby Temperature-Dependent NEXAFS Spectroscopy. The European Physical Journal B, 88, 13. http://dx.doi.org/10.1140/epjb/e2014-50481-9

[7] Kagoshima, S., Ishiguro, T. and Anzai, H. (1976) Observation of the Kohn Anomaly and the Peierls Transition in TTF-TCNQ by X-Ray Scattering. Journal of the Physical Society of Japan, 41, 2061. http://dx.doi.org/10.1143/JPSJ.41.2061

[8] Khanna, S.K., Pouget, J.P., Comes, R., Garito, A.F. and Heeger, A.J. (1977) X-Ray Studies of $2 \mathrm{k}_{\mathrm{F}}$ and $4 \mathrm{k}_{\mathrm{F}}$ Anomalies in Tetrathiafulvalene-Tetracyanoquinodimethane (TTF-TCNQ). Physical Review B, 16, 1468. http://dx.doi.org/10.1103/PhysRevB.16.1468

[9] Reitschel, H. (1973) The Giant Kohn Anomaly in a Peierls Semiconductor. Solid State Communications, $13,1859$.

[10] Bulaevskii, L.N. (1975) Peierls Structure Transition in Quasi-One-Dimensional Crystals. Soviet Physics Uspekhi, 18, 131. http://dx.doi.org/10.1070/PU1975v018n02ABEH001950

[11] Casian, A., Dusciac, V. and Coropceanu, Iu. (2002) Huge Carrier Mobilities Expected in Quasi-One-Dimensional Organic Crystals. Physical Review B, 66, Article ID: 165404. http://dx.doi.org/10.1103/PhysRevB.66.165404

[12] Casian, A. (2010) Violation of Wiedemann-Franz Law in Quasi-One-Dimensional Organic Crystals. Physical Review B, 81, Article ID: 155415. http://dx.doi.org/10.1103/PhysRevB.81.155415

[13] Sanduleac, I., Casian, A. and Pflaum, J. (2014) Thermoelectric Properties of Nanostructured Tetrathiotetracene Iodide 
Crystals in a Two-Dimensional Model. Journal of Nanoelectronics and Optoelectronics, 9, 247-252. http://dx.doi.org/10.1166/jno.2014.1574

[14] Casian, A. and Andronic, S. (2012) Phonons near Peierls Phase Transition in Quasi-One-Dimensional Organic Crystals. Proceedings of the 4th International Conference on Telecommunication, Electronics and Informatics, Chisinau, 17-20 May 2012, 258. (In Rumanian)

[15] Andronic, S. and Casian, A. (2013) Peierls Structural Transition in Quasi-One-Dimensional Organic Crystals. Modavian Journal of Physical Sciences, 12, 192.

[16] Andronic, S., Casian, A. and Dusciac, V. (2015) Peierls Structural Transition in Q1D Crystals of TTF-TCNQ Type for Different Values of Carrier Concentration. Materials Today: Proceedings, 2, 3829-3835. http://dx.doi.org/10.1016/j.matpr.2015.08.009

[17] Tiedje, T., et al. (1977) Temperature Dependence of Sound Velocity in TTF-TCNQ. Solid State Communications, 23, 713. http://dx.doi.org/10.1016/0038-1098(77)90478-1 